\title{
Multinacionales chinas: ¿cómo influyen los factores institucionales en sus patrones de localización?
}

\section{Chinese multinationals: how do institutional factors affect their location patterns?}

\author{
Diego Quer RAmón ${ }^{1}$ \\ Enrique Claver Cortés ${ }^{1}$ \\ LAURA RIENDA GARCía ${ }^{1}$ \\ Universidad de Alicante (España)
}

Recibido el 8 de abril de 2010 y aceptado el 21 de junio de 2010

$\mathrm{N}^{\circ}$ de clasificación JEL: F23

DOI: $10.5295 /$ cdg. $100216 \mathrm{dq}$

\section{Resumen:}

Este artículo analiza la influencia de diversos factores institucionales del país de destino sobre los patrones de localización de 29 grandes multinacionales chinas. A partir de una muestra de 127 decisiones de inversión directa en el exterior (IDE) en 52 países, nuestros resultados indican que una mayor dificultad a la hora de hacer negocios y un elevado riesgo político no condicionan sus decisiones de entrada. No obstante, la presencia de personas de etnia china en el país de destino, un mayor tamaño absoluto del mercado y un mayor volumen de exportaciones chinas hacia ese país influyen positivamente.

Palabras clave:

Multinacionales de países emergentes, China, factores institucionales, patrones de localización, inversión directa en el exterior.

\begin{abstract}
:
This paper analyses the impact of various host country institutional factors on the location patterns of 29 large Chinese multinationals. From a sample of 127 outward foreign direct investment (FDI) decisions made in 52 countries, our findings suggest that a greater difficulty in doing business and a high political risk in the host country do not discourage Chinese multinationals. However, the presence of overseas Chinese in the host country, a larger absolute host market size and a higher volume of Chinese exports to that country affect positively.
\end{abstract}

Keywords:

Emerging-market multinationals, China, institutional factors, location patterns, foreign direct investment.

\footnotetext{
${ }^{1}$ Universidad de Alicante, Departamento de Organización de Empresas, Campus de San Vicente del Raspeig, Ap. 99.E-03080. Alicante.Email: diego.quer@ua.es laura.rienda@ua.es enrique.claver@ua.es
} 


\section{INTRODUCCIÓN}

Que el centro de gravedad de la economía mundial se está desplazando hacia AsiaPacífico, en especial hacia China, es una realidad incuestionable. China ya es la segunda economía del mundo en términos de PIB, el mayor país exportador o el segundo mayor receptor de inversiones extranjeras (el primero, entre las economías emergentes). Además, en los últimos años, muchas empresas chinas se han lanzado a efectuar importantes inversiones en otros países. Como consecuencia, la inversión directa en el exterior (IDE) procedente de China se ha multiplicado por cuatro entre 2005 y 2009, convirtiendo al gigante asiático en el sexto mayor inversor mundial en 2009 (UNCTAD, 2010). Además, se estima que, hasta finales de 2009, ya había establecidas alrededor de 13000 empresas de capital chino en unos 177 países (MOFCOM, 2010).

La búsqueda de recursos (especialmente, recursos naturales), de mercados (en muchos casos, tratando de evitar restricciones a la exportación) o de activos estratégicos (en particular, tecnología, know how directivo o marcas reconocidas a nivel internacional) son las razones principales que están detrás de este espectacular crecimiento de la IDE china (Quer et al., 2007). Todo ello facilitado por las enormes reservas de divisas acumuladas gracias a las exportaciones, por el conocimiento adquirido cooperando con empresas extranjeras en China y, por supuesto, por el gobierno chino, que considera la expansión internacional de las empresas chinas como uno de los ejes fundamentales para el desarrollo económico del país (Hong y Sun, 2006).

A pesar de que este despegue de la IDE china ha sido relativamente reciente, operaciones como las adquisiciones de la división de PCs de IBM por parte de Lenovo, del fabricante británico de automóvil MG Rover por parte de Nanjing Automotive (actualmente fusionada con SAIC), la entrada en el capital del Standard Bank of Sudáfrica por parte de Industrial and Commercial Bank of China (ICBC) o la adquisición parcial de Repsol YPF Brasil por Sinopec, han provocado que las multinacionales chinas se estén situando en el punto de mira de economistas, políticos y observadores de todo el mundo.

La investigación en el campo de los negocios internacionales no ha sido ajena a este fenómeno (Buckley et al., 2007; Child y Rodrigues, 2005; Cui y Jiang, 2009; Matthews, 2006). Sin embargo, todavía existen determinados gaps, que hacen necesario profundizar en el conocimiento de las multinacionales chinas. En particular, todavía sabemos poco acerca de los factores que condicionan decisiones estratégicas clave en su proceso de internacionalización. Por ello, el objetivo de este trabajo es analizar la influencia que diversos factores institucionales tienen sobre una de las decisiones estratégicas más importantes que deben adoptar: la elección del país de destino. De forma más precisa, pretendemos estudiar cómo afectan al número de IDEs chinas en cada país tres factores concretos: el marco institucional para los negocios existente en el país de destino, su riesgo político y su distancia cultural respecto a China.

\section{TEORÍA INSTITUCIONAL Y MULTINACIONALES DE PAÍSES EMERGENTES}

La teoría institucional propone que las empresas efectúan sus elecciones estratégicas basadas en la interacción entre las instituciones y la propia organización, tratando de ob- 
tener legitimidad institucional respecto a los sistemas normativos y reguladores del país de destino. Esta perspectiva se ha erigido en los últimos años como uno de los marcos teóricos más adecuados para analizar decisiones estratégicas de empresas procedentes de economías emergentes (Peng et al., 2008).

Las multinacionales chinas, al igual que el resto, tienen que cumplir con los requerimientos institucionales de cada país (Rui y Yip, 2008). No obstante, en determinados destinos, se enfrentan a barreras institucionales superiores a las que deben afrontar empresas procedentes de otros países. Ello es debido a las reticencias que genera la propiedad estatal de muchas empresas chinas y la consiguiente posible búsqueda de objetivos no sólo comerciales (Globerman y Shapiro, 2009).

La fortaleza del marco institucional de un determinado país reduce los costes de hacer negocios para las empresas extranjeras. Por el contrario, su debilidad puede exagerar las asimetrías de información, por lo que las empresas afrontarán mayores riesgos y necesitarán gastar más recursos buscando información (Meyer et al., 2009). En un país donde no existan restricciones en el marco institucional, las empresas inversoras extranjeras no estarán sujetas a políticas discriminatorias relativas al porcentaje de propiedad en la subsidiaria, el acceso a recursos locales o la obligación de exportar una parte de la producción. Por tanto, proponemos la siguiente hipótesis:

Hipótesis 1: La fortaleza del marco institucional para los negocios en el país de destino afectará positivamente al número de IDEs chinas.

Otro de los factores institucionales que influye sobre las decisiones de entrada a un determinado país es su riesgo político. Las diferencias de riesgo político entre países afectan a la estabilidad de sus mercados, lo que condiciona a las empresas extranjeras que pretenden hacer negocios en ellos. El elevado grado de incertidumbre asociado a la propiedad extranjera o la mayor exposición de los activos ante una eventual expropiación son algunos de los factores que pueden obstaculizar las decisiones de IDE (Brouthers, 2002). Cabe esperar que las empresas multinacionales sean más reticentes a invertir en países con un entorno político inestable. En consecuencia, estos argumentos nos sugieren la siguiente hipótesis:

Hipótesis 2: Un mayor riesgo político en el país de destino afectará negativamente al número de IDEs chinas.

Por otra parte, las empresas multinacionales, cuando entran en un entorno institucional con diferente sistema normativo, deben cumplir con las expectativas sociales para construir una legitimidad social en el país de destino. La dificultad para obtener dicha legitimidad está relacionada con la distancia cultural entre los países de origen y de destino (Cui y Jiang, 2010). De hecho, la cultura puede ser considerada como parte de las instituciones informales del entorno, que apuntalan las instituciones formales (Peng et al., 2008). Además, la distancia cultural puede generar costes de obtención de información, perturbar los procesos de comunicación y dificultar la integración de la subsidiaria local, la aplicabilidad de las rutinas de la empresa o la adaptación del producto (Chen y Hu, 2002; Madhok, 1997). Por tanto, cabe esperar que:

Hipótesis 3: Una mayor distancia cultural entre China y el país de destino afectará negativamente al número de IDEs chinas. 


\section{EVIDENCIA EMPÍRICA}

La muestra para este estudio está formada por las IDEs efectuadas entre 2002 y 2008 por las 29 empresas chinas listadas en Fortune Global 500 (Fortune, 2008). Éste es un ranking anual de las 500 mayores empresas del mundo por volumen de facturación. Los datos sobre cada IDE fueron obtenidos a partir de las noticias publicadas en la web de China Daily (www.chinadaily.com.cn), el mayor periódico en lengua inglesa de China. Durante el periodo considerado, las 29 empresas chinas efectuaron 127 IDEs en 52 países, siendo ésta la muestra utilizada en nuestro estudio.

Los principales países de destino fueron Australia (10 IDEs), Indonesia y EE.UU. (9 IDEs), seguidos de Brasil, Reino Unido y Rusia (con 7 IDEs en cada uno). La tabla 1 recoge la distribución de las IDEs en función del nivel de desarrollo de los 52 países de destino (UNCTAD, 2010): países desarrollados (incluyendo a los miembros de la OCDE) y países en desarrollo. Siguiendo a Pangarkar y Yuan (2009), hemos incluido en la primera categoría a los países recientemente industrializados como Singapur. Se observa que la distribución de las IDEs chinas está bastante equilibrada entre ambos grupos, aunque predominan ligeramente las dirigidas a países en desarrollo, que representan casi un 52\% del total.

Tabla 1

Destinos de las IDEs de las grandes multinacionales chinas (2002-2008)

\begin{tabular}{|l|l|c|}
\cline { 2 - 3 } \multicolumn{1}{c|}{} & \multicolumn{1}{c|}{ PAÍSES } & $\mathrm{N}^{\circ}$ DE IDEs \\
\hline $\begin{array}{l}\text { Países desarrollados y } \\
\text { países recientemente } \\
\text { industrializados }\end{array}$ & $\begin{array}{l}\text { Alemania, Australia, Canadá, Chile, Corea del Sur, } \\
\text { EE.UU., España, Francia, Grecia, Holanda, Israel, } \\
\text { Italia, Japón, México, Noruega, Polonia, Reino Uni- } \\
\text { do, Singapur, Suiza }\end{array}$ & 61 \\
\hline Países en desarrollo & $\begin{array}{l}\text { Afganistán, Arabia Saudí, Argentina, Azerbaiyán, } \\
\text { Brasil, Congo (R.D.), Costa Rica, Ecuador, Emiratos } \\
\text { Árabes Unidos, Filipinas, India, Indonesia, Irán, } \\
\text { Jamaica, Jordania, Kazajstán, Mauritania, Mongolia, } \\
\text { Nigeria, Omán, Pakistán, Papua Nueva Guinea, Para- } \\
\text { guay, Perú, Rusia, Siria, Sudáfrica, Sudán, Ucrania, } \\
\text { Uzbekistán, Venezuela, Vietnam, Yemen }\end{array}$ & 66 \\
\hline Total & 52 & 127 \\
\hline
\end{tabular}

La empresa que efectuó un mayor número de IDEs durante el periodo analizado fue Noble Group (21 IDEs), conglomerado con presencia en sectores como logística, minería, agricultura o energía. La segunda fue la petrolera CNPC (15 IDEs). En tercer lugar aparece Hutchison Whampoa (11 IDEs), presente en varios sectores (comercio, inmobiliario, telecomunicaciones y servicios portuarios). A continuación encontramos a la petrolera Sinopec (9 IDEs), al Bank of China (también 9 IDEs) y a la informática Lenovo (7 IDEs).

La tabla 2 presenta la distribución sectorial de las empresas de nuestra muestra. Como puede observarse, casi una cuarta parte de las IDEs ha sido efectuada en el sector de pe- 
tróleo y gas (30 IDEs). Este hecho denota la todavía importante dependencia exterior de China para obtener recursos naturales que permitan sostener su crecimiento económico. En línea similar se encontrarían las 16 IDEs efectuadas en el sector del metal. No obstante, resultan también destacables las inversiones en el sector bancario (17) y de telecomunicaciones (11), reflejo de que la búsqueda de mercados o de activos estratégicos está empezando a ganar terreno como objetivo de la expansión internacional de las multinacionales chinas.

Tabla 2

Distribución sectorial de las IDEs de las grandes multinacionales chinas (2002-2008)

\begin{tabular}{|l|c|}
\hline \multicolumn{1}{|c|}{ SECTOR } & $\mathrm{N}^{\circ}$ de IDEs \\
\hline Extracción de petróleo y gas & 30 \\
\hline Banca & 17 \\
\hline Metal & 16 \\
\hline Telecomunicaciones & 11 \\
\hline Servicios portuarios & 7 \\
\hline Informática & 7 \\
\hline Productos químicos & 6 \\
\hline Productos alimenticios & 5 \\
\hline Vehículos & 5 \\
\hline Construcción & 5 \\
\hline Derivados del petróleo & 4 \\
\hline Comercio al por mayor & 3 \\
\hline Comercio al por menor & 3 \\
\hline Transporte marítimo & 3 \\
\hline Electricidad & 2 \\
\hline Seguros & 1 \\
\hline Intermediación financiera & 1 \\
\hline Inmobiliario & 1 \\
\hline Total & 127 \\
\hline
\end{tabular}

Para comprobar empíricamente las ideas propuestas anteriormente en nuestro marco teórico, hemos aplicado una regresión lineal. Este modelo permite examinar el impacto de una serie de variables independientes sobre una variable dependiente. La variable dependiente en nuestro caso es el número de IDEs chinas en cada país, mientras que las variables independientes se refieren a los tres factores institucionales vinculados al país de destino explicados anteriormente.

La primera es la fortaleza del marco institucional para los negocios en el país de destino, que se ha medido a partir del ranking que elabora anualmente el Banco Mundial según la facilidad para hacer negocios en cada país (World Bank, 2008). A partir de una serie de variables que condicionan la actividad empresarial, este informe anual clasifica 181 economías, desde la de mayor facilidad para los negocios (1) hasta la de mayor dificultad (181). 
La segunda variable independiente es el riesgo político del país de destino, aproximado a partir de la clasificación ofrecida por la Guía Internacional Riesgo País (PRS, 2009). En función de diversos factores que afectan al riesgo político, esta guía asigna una puntuación a los diversos países, con la salvedad de que una menor puntuación indica un mayor riesgo político, mientras que una mayor puntuación denota un menor riesgo. La tercera variable independiente es la distancia cultural entre China y cada país de destino, para la cual hemos empleado dos medidas. La primera es el índice de distancia cultural elaborado por Kogut y Singh (1988), a partir de las dimensiones culturales de Hofstede (1980): distancia al poder, evitación de la incertidumbre, individualismo y valores predominantes. Combinando las puntuaciones para estas cuatro dimensiones, el índice proporciona una medida cuantitativa de la distancia cultural. Para cada país, una mayor puntuación implica una mayor distancia cultural respecto a China. Como segunda medida de la distancia cultural se ha utilizado el porcentaje de personas de etnia china en cada país de destino (Ohio University, 2009).

Además de las tres variables independientes anteriores, hemos incluido otras variables que también pueden condicionar las decisiones de localización de las multinacionales chinas. Por un lado, hemos incorporado tres variables relativas a las características del mercado de destino (UN Statistics Division, 2009): tamaño absoluto (aproximado a partir del PIB del país de destino), tamaño relativo (PIB per cápita) y tasa de crecimiento (porcentaje de crecimiento anual del PIB). Por otra parte, la decisión de una empresa china de efectuar una IDE en un país concreto también podría verse afectada por la intensidad de las relaciones comerciales entre China y dicho país de destino. Por ello, hemos incluido otras dos variables (MOFCOM, 2009): exportaciones chinas a cada país e importaciones chinas desde cada país.

La regresión lineal para el contraste de hipótesis se ha efectuado empleando el software estadístico SPSS 16.0. La tabla 3 recoge la matriz de correlaciones entre las variables incorporadas al modelo.

Tabla 3

Matriz de correlaciones

\begin{tabular}{|l|c|c|c|c|c|c|c|c|}
\cline { 2 - 11 } \multicolumn{1}{c|}{} & 1 & 2 & 3 & 4 & 5 & 6 & 7 & 8 \\
\hline 1. Facilidad para hacer negocios & & & & & & & & \\
\hline 2. Riesgo político & -0.77 & & & & & & & \\
\hline 3. Distancia cultural (índice) & -0.48 & 0.56 & & & & & & \\
\hline 4. Distancia cultural (personas de etnia china) & -0.01 & 0.13 & -0.10 & & & & & \\
\hline 5. Tamaño absoluto del mercado & -0.51 & 0.51 & 0.48 & -0.04 & & & & \\
\hline 6. Tamaño relativo del mercado & -0.73 & 0.79 & 0.68 & -0.06 & 0.73 & & & \\
\hline 7. Tasa de crecimiento del mercado & 0.43 & -0.56 & -0.45 & 0.04 & -0.45 & -0.55 & & \\
\hline 8. Exportaciones chinas al país de destino & -0.40 & 0.32 & 0.27 & -0.05 & 0.58 & 0.41 & -0.41 & \\
\hline 9. Importaciones chinas desde el país de destino & -0.37 & 0.28 & 0.18 & -0.01 & 0.51 & 0.35 & -0.33 & 0.70 \\
\hline $\begin{array}{l}N^{\circ} \text { de observaciones: 52 } \\
\text { Las correlaciones por encima de /0.32/ son significativas con } \mathrm{p}<0.05 \\
\text { Las correlaciones por encima de /0.37/ son significativas con } \mathrm{p}<0.01 \\
\text { El FIV (factor de inflación de la varianza) más elevado es } 6.31\end{array}$ \\
\hline
\end{tabular}


La tabla 4 expone los resultados del análisis de regresión.

Tabla 4

Análisis de regresión: influencia de los factores institucionales sobre la localización de las multinacionales chinas

\begin{tabular}{|l|c|}
\hline \multicolumn{2}{|c|}{ VARIABLE DEPENDIENTE: número de IDEs chinas en cada país } \\
\hline \multicolumn{1}{|c|}{ VARIABLES INDEPENDIENTES } & COEFICIENTE $\beta$ \\
\hline Facilidad para hacer negocios & 0.06 \\
\hline Riesgo político & -0.07 \\
\hline Distancia cultural (índice) & -0.13 \\
\hline Distancia cultural (personas de etnia china) & $0.40^{* *}$ \\
\hline Tamaño absoluto del mercado & $0.42^{*}$ \\
\hline Tamaño relativo del mercado & 0.08 \\
\hline Tasa de crecimiento del mercado & -0.10 \\
\hline Exportaciones chinas al país de destino & $0.39 \dagger$ \\
\hline Importaciones chinas desde el país de destino & -0.20 \\
\hline $\begin{array}{l}\mathrm{N}^{\circ} \text { de observaciones: } 52 \\
\mathrm{R}^{2}(\% \text { de varianza explicado): } 0.49\end{array}$ \\
$\begin{array}{l}\mathrm{F}\left(\text { significación del modelo): } 3.40^{* *}\right. \\
\dagger \mathrm{p}<0.10, * \mathrm{p}<0.05, * * \mathrm{p}<0.01\end{array}$ \\
\hline
\end{tabular}

Los resultados no ofrecen significación estadística para la relación positiva entre la fortaleza de las instituciones que facilitan realizar negocios en el país de destino y el número de IDEs chinas en ese país. Una posible explicación para este resultado, contrario al inicialmente previsto, puede radicar en que aunque algunas empresas chinas no tengan todavía ventajas competitivas basadas en tecnología o marca, sí disponen de habilidad para manejar relaciones en un entorno complejo como China. Esto les proporciona una ventaja sobre las multinacionales de países desarrollados a la hora de efectuar IDEs en destinos con esas características institucionales menos favorables (Morck et al., 2008). De hecho, la similitud entre los entornos institucionales de dos países puede permitir a la dirección de la empresa organizar un mercado interno de forma más eficiente que entre dos países con entornos institucionales muy diferenciados (Henisz, 2003).

La relación negativa entre riesgo político en el país de destino e IDE china tampoco ha podido ser confirmada. Es posible apuntar varias explicaciones para este resultado que contradice la influencia convencional del riesgo político. En primer lugar, el tamaño de la IDE puede condicionar el impacto del riesgo político. Así, en el caso de efectuar grandes inversiones, las empresas chinas pueden aprovechar la oportunidad de adquirir activos más baratos en países con un sistema político inestable (Malhotra y Zhu, 2009). En segundo lugar, las empresas chinas pueden tratar de aprovechar las oportunidades que presentan los países de alto riesgo, cuyos mercados pueden estar poco explotados o incluso ignorados 
por las grandes multinacionales occidentales (Bunyaratavej y Hahn, 2007). En tercer lugar, una parte importante de la IDE china se ha dirigido a países con los que China mantiene estrechos vínculos políticos e ideológicos, muchos de los cuales presentan un elevado riesgo político (Buckley et al., 2007).

En cualquier caso, debe tenerse en cuenta que existen distintos niveles de riesgo asociados al país de destino (Jiménez y Durán, 2009). Por un lado, existe un nivel macro, que asumen todos los inversores extranjeros, así como un nivel meso-económico, derivado de la diferente sensibilidad de los sectores al riesgo de cada país. Además, empresas de un mismo sector pueden asumir diferenciales de riesgo más favorables en un mismo país, lo que sugiere que esta clase de riesgo puede gestionarse si las empresas desarrollan habilidades políticas y de negociación, especialmente en entornos institucionales escasamente desarrollados y con elevado poder discrecional del gobierno.

En este contexto, cabe recordar que una parte importante de las IDEs chinas de nuestra muestra se han dirigido a sectores regulados, como petróleo y gas, banca, telecomunicaciones o servicios públicos. En general, estos sectores regulados presentan tres características (Henisz, 2003): el gobierno desempeña un papel central como proveedor o supervisor; existe necesidad de capital extranjero, lo que fuerza al gobierno local a abrir el sector a la iniciativa privada; y hay idiosincrasias institucionales, que dificultan a las instituciones financieras internacionales valorar los créditos así como a los inversores cubrir sus riesgos con instrumentos financieros.

La investigación reciente sobre expansión internacional en estos sectores regulados reta la noción de que los países con una elevada inestabilidad política son poco atractivos para las empresas extranjeras (García-Canal y Guillén, 2008). En el momento de entrar, las empresas de estos sectores pueden preferir países donde el poder ejecutivo del gobierno no esté restringido por los poderes legislativo y judicial. Esa mayor discrecionalidad del gobierno puede permitir que las empresas inversoras consigan un trato preferente. En otras palabras, es necesario considerar la habilidad de cada empresa para manejar las idiosincrasias institucionales e influir sobre los gobiernos e instituciones del país de destino (Henisz, 2003).

Respecto a la pronosticada influencia negativa de la distancia cultural sobre la IDE china, nuestros resultados han podido confirmarla parcialmente. Utilizando como aproximación el índice de distancia cultural (Kogut y Singh, 1988), la influencia negativa no resulta estadísticamente significativa. Un posible argumento para justificar este resultado sería que la influencia de la distancia cultural puede depender de los objetivos perseguidos por las multinacionales chinas. Mientras que las IDEs chinas que buscan mercados pueden ir inicialmente dirigidas a países más cercanos culturalmente, las que buscan know-how suelen ir dirigidas a países desarrollados de Norte América y Europa, culturalmente más distantes (Young et al., 1996). Además, es probable que muchas empresas chinas no rehuyan tanto de la distancia cultural, gracias a las alianzas que desde hace años mantienen en la propia China con multinacionales de países desarrollados (Luo y Tung, 2007).

Sin embargo, empleando como medida de proximidad cultural la proporción de personas de etnia china en el país de destino, sí observamos una influencia positiva en línea con lo esperado. Los contactos y redes sociales (conocidos en China como guanxi) constituyen para los chinos uno de los ingredientes esenciales a la hora de hacer negocios. Por tanto, la presencia de personas de etnia china en un determinado país puede reducir los riesgos y 
costes asociados con la identificación de oportunidades de negocio por parte de las empresas chinas (Buckley et al., 2007).

Nuestros resultados indican que otras dos variables también condicionan las decisiones de localización de las multinacionales chinas. El número de IDEs chinas en un país está asociado positivamente con el tamaño absoluto del mercado de destino y con el volumen de exportaciones chinas hacia dicho país. Ambos efectos sugieren que el objetivo de buscar y mantener mercados puede estar detrás de dicha relación. Además, las empresas chinas afrontan restricciones cuantitativas a la exportación a determinados países, en ocasiones incluso superiores a las que deben soportar otras empresas. Por ello, la IDE para fabricar en esos destinos puede resultar una buena alternativa para poder seguir accediendo a dichos mercados (Hong y Sun, 2006).

\section{CONCLUSIONES}

A partir del enfoque institucional, especialmente adecuado para analizar la expansión internacional de las empresas de economías emergentes, hemos pretendido analizar la influencia de diversos factores institucionales del país de destino sobre las decisiones de localización de las multinacionales chinas. En nuestra opinión, este artículo ofrece algunas contribuciones interesantes.

Por un lado, nuestro estudio ha puesto de manifiesto que los patrones de localización seguidos por las multinacionales chinas presentan algunas características acordes con los planteamientos tradicionales del enfoque institucional y otras menos convencionales. Así, la presencia de personas de etnia china en el país de destino parece ser un factor que ayuda a las empresas chinas a superar la posible barrera cultural. Asimismo, un mayor tamaño absoluto del mercado de destino constituye un factor de localización atractivo, en particular para aquellas empresas chinas que busquen mercados en su expansión internacional. Este resultado resulta congruente con el hecho de que un mayor volumen de exportaciones chinas hacia ese mercado esté relacionado positivamente con la IDE china.

Sin embargo, otros resultados obtenidos parecen desviarse de la lógica convencional observada en las decisiones de localización adoptadas por multinacionales procedentes de otros países, en especial occidentales. Un débil marco institucional en el país de destino, caracterizado por una mayor dificultad para hacer negocios y un elevado riesgo político, no desincentiva a las multinacionales chinas. Además, utilizando una medida convencional ampliamente empleada en la literatura sobre empresas multinacionales, no hemos podido confirmar que la distancia cultural constituya una barrera institucional significativa para las empresas chinas. Todo ello puede suponer un reto para los planteamientos tradicionales del enfoque institucional, que necesitarían una adaptación para explicar el comportamiento internacional de las multinacionales chinas.

Por otra parte, la principal contribución empírica de nuestra investigación radica en haber aportado nueva evidencia basada en el enfoque institucional sobre un fenómeno que está adquiriendo una importancia económica creciente: la irrupción internacional de multinacionales procedentes de mercados emergentes. Aunque en los últimos años están apareciendo cada vez más trabajos centrados en las multinacionales chinas, dado el carácter reciente de su proceso de internacionalización, este tópico todavía se encuentra en una 
etapa inicial de consolidación. Por tanto, se hace necesario profundizar en el conocimiento de un tipo de multinacionales que están llamadas a jugar un rol cada vez más relevante en los negocios internacionales.

En esta línea, nuestra aportación empírica tiene implicaciones desde el punto de vista de la gestión, tanto pública como privada. Por un lado, los responsables de la administración pública en cada país pueden tener un punto de referencia para el diseño de políticas tendentes a manejar sus relaciones bilaterales con el gigante asiático, en función de la convergencia o divergencia de intereses. Así, las adquisiciones por parte china de empresas en dificultades de países desarrollados y su posterior relanzamiento pueden contribuir a evitar pérdidas de empleo, algo que podría ser bien recibido por los gobiernos anfitriones. De forma análoga, la inversión china en países ricos en materias primas puede ayudar a revitalizar sectores en declive e incluso incrementar la exportación de esos países. No obstante, la propiedad estatal de muchas de estas multinacionales chinas también puede provocar recelos en aquellos países donde los activos que buscan son considerados estratégicos. En ese caso, cabe esperar obstáculos y medidas proteccionistas.

Por otra parte, los directivos de empresas, especialmente en países occidentales desarrollados, no pueden ser ajenos a este fenómeno emergente dado que, como ya hemos comentado anteriormente, las multinacionales chinas se están posicionando en sectores cada vez más intensivos en tecnología y conocimiento, y han sido capaces de hacerse con el control de empresas emblemáticas. Por consiguiente, conocer sus patrones de localización resulta indispensable, ya sea para protegerse de su competencia o para buscar alianzas estratégicas acompañándolas en su proceso de internacionalización. Este es el caso de la alianza protagonizada por la española Telefónica y la multinacional china Huawei, uno de los líderes mundiales en fabricación de equipos de redes y telecomunicaciones, o también de la ya mencionada entrada de la petrolera china Sinopec en el capital de la filial brasileña de Repsol.

En cualquier caso, nuestros resultados deben ser interpretados teniendo en cuenta las limitaciones inherentes a la naturaleza de nuestro estudio empírico. La principal tal vez sea el hecho de no haber podido incorporar algunas variables específicas que pueden condicionar también las decisiones de localización, como el motivo concreto de cada decisión de IDE: búsqueda de recursos, de mercados o de activos estratégicos. Dependiendo de cuál sea el objetivo perseguido por las empresas chinas, los factores institucionales vinculados a dicha localización pueden jugar un papel distinto. Por ejemplo, las restricciones institucionales que pueden surgir cuando la empresa efectúa una IDE para acceder a un recurso considerado estratégico para el país de destino pueden no aparecer cuando se invierte en el mismo país con la finalidad de acceder a su mercado.

Ello deja abiertas varias vías interesantes para la investigación futura. Además de la incorporación de esas otras variables que pueden condicionar los patrones de localización, existen otras cuestiones que requieren investigación adicional. Resultaría interesante analizar cómo adoptan las empresas chinas otras decisiones importantes en su aventura internacional, como la elección del modo de entrada o del tipo de personal nativo o expatriado para sus subsidiarias exteriores. Profundizar en las similitudes y diferencias entre este reciente proceso de internacionalización de las multinacionales chinas y el que protagonizaron años atrás sus vecinas asiáticas procedentes de Japón o Corea del Sur puede ser otra prometedora vía de investigación futura. 


\section{REFERENCIAS BIBLIOGRÁFICAS}

BROUTHERS, K.D. (2002): "Institutional, cultural and transaction cost influences on entry mode choice and performance", Journal of International Business Studies, Vol. 33, No. 2, pp. 203-221.

BUCKLEY, P,J.; CLEGG, L.J.; CROSS, A.R.; LIU, X.; VOSS, H. Y ZHENG, P. (2007): "The determinants of Chinese foreign direct investment", Journal of International Business Studies, Vol. 38, No. 4, pp. 499-518.

BUNYARATAVEJ, K. Y HAHN, E.D. (2007): "Outward foreign direct investments from China: Theory and determinants", AIB 2007 Annual Meeting, Indianapolis.

CHEN, H. Y HU, M.Y. (2002): "An analysis of entry mode and its impact on performance", International Business Review, Vol. 11, No. 2, pp. 193-210.

CHILD, J. Y RODRIGUES, S.B. (2005): "The internationalization of Chinese firms: A case for theoretical extension?", Management and Organization Review, Vol. 1, No. 3, pp. 381-410.

CUI, L. Y JIANG, F. (2009): "FDI entry mode choice of Chinese firms: A strategic behavior perspective", Journal of World Business, Vol. 44, No. 4, pp. 434-444.

CUI, L. Y JIANG, F. (2010): "Behind ownership decision of Chinese outward FDI: Resources and institutions", Asia Pacific Journal of Management, Vol. 27, № 4, pp. 751-774.

FORTUNE (2008): Fortune Global 500 (2008 edition), New York, Time Inc.'s Fortune/Money Group.

GARCÍA-CANAL, E. Y GUILLÉN, M.F. (2008): "Risk and the strategy of foreign location choice in regulated industries", Strategic Management Journal, Vol. 29, No. 10, pp. 1097-1115.

GLOBERMAN, S. Y SHAPIRO, D. (2009): "Economic and strategic considerations surrounding Chinese FDI in the United States", Asia Pacific Journal of Management, Vol. 26, No. 1, pp. 163-183.

HENISZ, W.J. (2003): "The power of the Buckley and Casson thesis: The ability to manage institutional idiosyncrasies", Journal of International Business Studies, Vol. 34, No. 2, pp. 173-184.

HOFSTEDE, G. (1980): Culture's consequences. International differences in work-related values, Newbury Park, Sage Publications.

HONG, E. Y SUN, L. (2006): "Dynamics of internationalization and outward investment: Chinese corporations' strategies”, The China Quarterly, No. 187, pp. 610-634.

JIMÉNEZ, A. Y DURÁN, J.J. (2009): "La responsabilidad social de la empresa multinacional española frente al riesgo político de los países de destino: ¿Amenaza u oportunidad?", Revista de Responsabilidad Social de la Empresa, No. 3, pp. 131-158.

KOGUT, B. Y SINGH, H. (1988): "The effect of national culture on the choice of entry mode", Journal of International Business Studies, Vol. 19, No. 3, pp. 411-432.

LUO, Y. Y TUNG, R.L. (2007): "International expansion of emerging market enterprises: A springboard perspective", Journal of International Business Studies, Vol. 38, No. 4, pp. 481-498.

MADHOK, A. (1997): "Cost, value and foreign market entry mode: The transaction and the firm", Strategic Management Journal, Vol. 18, No. 1, pp. 39-61.

MALHOTRA, S. Y ZHU, P.C. (2009): "Determinants and valuation impact of cross-border acquisitions by firms from China and India", AIB 2009 Annual Meeting, San Diego.

MATHEWS, J.A. (2006): "Dragon multinationals: New players in $21^{\text {st }}$ century globalization", Asia Pacific Journal of Management, Vol. 23, No. 1, pp. 5-27.

MEYER, K.E.; ESTRIN, S.; BHAUMIK, S.K. Y PENG, M.W. (2009): "Institutions, resources, and entry strategies in emerging economies", Strategic Management Journal, Vol. 30, No. 1, pp. 61-80.

MOFCOM (2010): 2009 Statistical bulletin of China's outward foreign direct investment, Beijing, Ministry of Commerce.

MOFCOM (2009): Total import \& export value by country (region), Beijing, Ministry of Commerce. 
MORCK, R.; YEUNG, B. Y ZHAO, M. (2008): "Perspectives on China's outward foreign direct investment", Journal of International Business Studies, Vol. 39, No. 3, pp. 337-350.

OHIO UNIVERSITY (2009): Distribution of the ethnic Chinese population around the world, Ohio University Library and the Shao Center.

PANGARKAR, N. Y YUAN, L. (2009): "Location in internationalization strategy: Determinants and consequences", Multinational Business Review, Vol. 17, No. 2, pp. 37-68.

PENG, M.W.; WANG, D.Y.L. Y JIANG, Y. (2008): "An institution-based view of international business strategy: A focus on emerging economies", Journal of International Business Studies, Vol. 39, No. 5, pp. 920-936.

PRS (2009): International Country Risk Guide (ICRG), The Political Risk Services Group.

QUER, D.; CLAVER, E. Y RIENDA, L. (2007): "Multinacionales chinas. El nuevo reto del escenario global”, Boletín Económico de ICE, No. 2925, pp. 11-24.

RUI, H. Y YIP, G.S. (2008): "Foreign acquisitions by Chinese firms: A strategic intent perspective", Journal of World Business, Vol. 43, No. 2, pp. 213-226.

UN STATISTICS DIVISION (2009): National accounts main aggregates database, United Nations Statistics Division.

UNCTAD (2010): World Investment Report 2010. Investing in a low-carbon economy, New York and Geneva, UNCTAD.

WORLD BANK (2008): Doing Business 2009, Washington, The World Bank.

YOUNG, S.; HUANG, C.H. Y MCDERMOTT, M. (1996): "Internationalization and competitive catch-up processes: Case study evidence on Chinese multinational enterprises", Management International Review, Vol. 36, No. 4, pp. 295-314. 